\title{
Unusual presentation of Endocervical Adenocarcinoma following hysterectomy for high grade CGIN
}

Thomas Hall, Mr. Andrew Phillips, Gerhard Van Schalkwyk, Mr. Anish Bali.

\section{Institutional affiliations}

University of Nottingham Medical School, Nottingham, NG7 2UH

University Hospitals of Derby and Burton NHS Foundation Trust, Derby, DE22 3NE

University Hospitals of Derby and Burton NHS Foundation Trust, Derby, DE22 3NE

University Hospitals of Derby and Burton NHS Foundation Trust, Derby, DE22 3NE

\section{Corresponding author}

Mr Thomas Hall, University of Nottingham, University Park ,Nottingham United Kingdom NG7 2RD

Email:mzytgha@nottingham.ac.uk

Mr Andrew Phillips University Hospitals of Derby and Burton NHS Foundation Trust, Department of Obstetrics and Gynaecology, Uttoxeter Road DERBY United Kingdom DE22 3NE

Email: andrew.phillips6@nhs.net

Received Date: May 01, 2020

Accepted Date: May 18, 2020

Published Date: Jun 13, 2020

\section{Introduction:}

Cervical carcinoma is the fourteenth most common cancer in the UK with 1 in every 142 women being diagnosed (1). Cervical carcinoma can be subdivided into numerous histological types, of which endocervical adenocarcinoma, represents $20-25 \%$ of all cancers (2). The proportion of cervical cancers diagnosed as endocervical adenocarcinomas has increased as the introduction of the cervical screening for squamous precancerous lesions has reduced the number of squamous cell cancers of the cervix. The most common subtype of endocervical adenocarcinoma is associated with human papillomavirus (HPV) type 18 (3). Often these cancers have a diffuse growth pattern and are closely associated with CGIN (adenocarcinoma in situ) making measurement of the extent of disease difficult. Cervical adenocarcinomas are staged according to the (FIGO) International Federation of Gynaecology and Obstetrics) system. Early stage cervical cancer is usually treated increasing radicality of surgical excision whilst advanced cervical cancer is treated with radiotherapy and chemotherapy.

\section{Case Presentation:}

A 52-year-old woman with a history of bilateral breast cancer and a previous large loop excision of transformation zone (LLETZ) for glandular dyskaryosis; presented with a recent abnormal cervical smear highlighting endocervical changes and an increase in vaginal discharge. At colposcopy an area of abnormality was seen that extended into the endocervical canal. This was treated with a second LLETZ that on histological review demonstrated incompletely excised high-grade cervical glandular intra-epithelial neoplasia (CGIN) and involvement of the endocervical margin. In view of her two treatments and incompletely excised disease the colposcopy multidisciplinary team (MDT) suggested a complete total laparoscopic hysterectomy and bilateral salpingo oophorectomy (TLH-BSO).

From this, an incidental diagnosis of endocervical adenocarcinoma with myometrial stroma invasion of $2 \mathrm{~mm}$ but with superficial spread over the entirety of the uterine endometrium was made. This was staged according to the FIGO system of the time as stage 1B1, an unanticipated presentation of cervical cancer.

\section{Investigations:}

The case was discussed in the gynaecological cancer MDT recommended that a CT scan and PET scan were to be performed. No pathological nodal disease or distant metastasis was identified. The patient was offered either completion nodal staging or adjuvant radiotherapy. The patient opted for laparoscopic removal of pelvic and para-aortic lymph nodes over radiotherapy which were negative for metastasis and thus no further treatment was required.

Treatment / Outcome and Follow Up

This patient was followed up according to the cancer centre protocol. With clinical followup in clinic every 3 months for the first year, 4 months for the second year and 6 months for the 3 years thereafter. Additional MRIs were to be undertaken at 6 and 18 months. At 12 months after diagnosis is patient is well with no signs of recurrent disease.

\section{Discussion:}

This is an endocervical adenocarcinoma that developed on the background of high-grade CGIN. It also showed extensive colonisation of the endometrium of the lower uterine segment and the whole endometrium. There was diffuse block type immunoreactivity with p16. CEA was diffusely positive. ER,WT-1, and vimentin were largely negative. This is in keeping with the usual type (HPV-related) adenocarcinoma.

Measurement of the tumour was difficult as there was extensive highgrade CGIN and the tumour did not exhibit a stromal desmoplastic response. However, on the basis of the lateral spread, the tumour was best regarded as Figo stage 1B1 (the depth of invasion was quite shallow with the tumour confined to the inner half of the cervical stroma). The colonisation of the endometrium did not influence the staging.

\section{Patient's Perspective:}

The patient stated that she was delighted that her story would be shared with the Gynaecological Community. She found the whole experience to be upsetting due to unusual and unforeseen presentation of her disease, but was delighted with the treatment and follow-up that she received.

\section{Acknowledgements: None}

Funding: Not applicable

Availability of data and materials: Not applicable

Authors' contributions

- Data collection: TH

- Manuscript preparation $\mathrm{TH} / \mathrm{AP} / \mathrm{GvS} / \mathrm{AB}$

- $\quad$ Concept AP

Ethics approval and consent to participate: Retrospective review, written consent obtained by the patient for publication.

Patient consent for publication: Written consent obtained by the patient 


\section{Journal of Clinical Obstetrics and Gynecology Research}

for publication

Competing interests: None

Authors' information (optional): Not applicable

\section{References:}

Cancer Research UK, www.cancerresearchuk.org/health-professional/ cancer-statistics/statistics-by-cancer-type/cervical-cancer, Accessed [February] [2019].
Solomon D, Breen N, McNeel T. Cervical cancer screening rates in the United States and the potential impact of implementation of screening guidelines. CA: a cancer journal for clinicians. 2007 Mar;57(2):105-11.

Lima PC, Teixeira J, Aires GN, Andrade LD. Endocervical gastric-type adenocarcinoma, an unrelated HPV tumour: difficulties in screening and diagnosis. BMJ case reports. 2017 Apr 11;2017:bcr-2017. 